\title{
QUALITY LOCAL REFINEMENT OF TETRAHEDRAL MESHES BASED ON 8-SUBTETRAHEDRON SUBDIVISION
}

\author{
ANWEI LIU AND BARRY JOE
}

\begin{abstract}
Let $\mathcal{T}$ be a tetrahedral mesh. We present a 3-D local refinement algorithm for $\mathcal{T}$ which is mainly based on an 8-subtetrahedron subdivision procedure, and discuss the quality of refined meshes generated by the algorithm. It is proved that any tetrahedron $\mathbf{T} \in \mathcal{T}$ produces a finite number of classes of similar tetrahedra, independent of the number of refinement levels. Furthermore, $\eta\left(\mathbf{T}_{i}^{n}\right) \geq c \eta(\mathbf{T})$, where $\mathbf{T} \in \mathcal{T}, c$ is a positive constant independent of $\mathcal{T}$ and the number of refinement levels, $\mathbf{T}_{i}^{n}$ is any refined tetrahedron of $\mathbf{T}$, and $\eta$ is a tetrahedron shape measure. It is also proved that local refinements on tetrahedra can be smoothly extended to their neighbors to maintain a conforming mesh. Experimental results show that the ratio of the number of tetrahedra actually refined to the number of tetrahedra chosen for refinement is bounded above by a small constant.
\end{abstract}

\section{INTRODUCTION}

The solution of some types of partial differential equations (PDEs) using the finite element method is an adaptive process, which in general consists of mesh generation, solution of linear equations, error estimation, and mesh refinement. In the mesh refinement step, varying element sizes are used to improve the solution of PDEs, and an efficient scheme, local refinement, is adopted to locally refine some regions where approximation errors are unacceptable. Also, the adaptive mesh refinement technique is needed in the multigrid method, in which the solution of a problem is obtained by alternately solving the problem on several levels of coarse to fine grids. It is desirable that the adaptive refinement produces refined elements of good shape using a fast refining process. These are the goals of our 3-D local refinement algorithm (LRA).

Not many results have been published on the quality of meshes produced by $3-\mathrm{D}$ local refinement algorithms (LRAs), although some 2-D quality LRAs are available $[17,18,19,2,1,13]$ and have proved to be successful in adaptive finite element analysis [14]. Some 3-D LRAs are used in practice without theoretical analysis on the quality of the refined meshes [16, 20]. In the bisection method of [8], the longest edge is always chosen to be bisected and diameter bounds are given for simplices of any dimension. However, whether the shapes of subtetrahedra produced by repeated longest-edge bisection degrade arbitrarily is still an open problem. In [12], we presented an LRA for tetrahedral meshes based on a special bisection

Received by the editor May 28, 1994 and, in revised form, July 5, 1995.

1991 Mathematics Subject Classification. Primary 65N50; Secondary 51M20, 52B10, 65M50.

This work was partially supported by a grant from the Natural Sciences and Engineering Research Council of Canada. 
procedure, which is the first theoretically guaranteed-quality LRA for tetrahedral meshes based on bisection. In this paper, we present a quality LRA based on an 8-subtetrahedron subdivision procedure, which is an extension of the 2-D LRA in $[2,1,13]$. The 8 -subtetrahedron subdivision may be preferable to bisection if the initial mesh is relatively coarse and needs to be refined quickly (this is also the case in the multigrid method, since the grids at two consecutive levels should in general be significantly different), because the volume of subtetrahedra in 8-subtetrahedron subdivision decreases faster than in bisection.

We consider a local refinement process that includes two major steps. First, a set $S$ of basic elements is chosen from an existing mesh $\mathcal{T}$ according to numerical results and error estimates from previous computations. Next, each element in $S$ is refined, and a procedure is needed to keep the final mesh conforming, where a conforming mesh is one in which the intersection of any two tetrahedra $\mathbf{T}_{1}, \mathbf{T}_{2}$ of the mesh is either a common face of $\mathbf{T}_{1}$ and $\mathbf{T}_{2}$, or a common edge, or a common vertex, or empty.

The basic idea in [1] is as follows. Normally, a triangle is regularly refined by dividing it into four similar triangles, as illustrated in Figure 1a. If the triangle has an interior angle greater than $\pi / 2$, then it is regularly refined into two similar triangles, and two geometrically better triangles, as illustrated in Figure 1b. At the boundary of a refined region, it is necessary to divide a triangle into only two triangles, called "green" triangles, by inserting an edge, as illustrated in Figure 1c. Refinement into green triangles is done only as a temporary measure; at each step of the adaptive process, if the green triangles need to be further refined, then these triangles are removed and the parent elements are regularly refined. Obviously, the degradation of geometry in this approach is bounded, since the green triangles are never further refined.

Likewise, in 3-D, we want to use a regular refinement called 8-subtetrahedron subdivision, which is defined as follows. Suppose each triangular face of a tetrahedron $\mathbf{T}$ is refined into four similar subtriangles by connecting the midpoints of the edges (see Figure 1a) as shown in Figure 2. Then we obtain four similar subtetrahedra at the four corners and an octahedron in the interior. By adding an interior edge, called the center edge, $\mathbf{t}_{02} \mathbf{t}_{13}$ say, in the middle of the octahedron, $\mathbf{T}$ is subdivided into eight subtetrahedra. We use $\mathrm{SUB}_{8}$ to denote the 8-subtetrahedron subdivision described here.

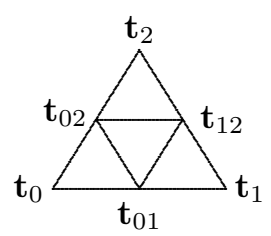

(a)

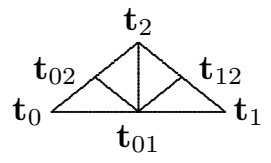

(b)

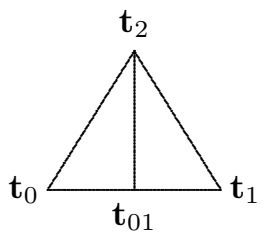

(c)

FiguRE 1. Illustration for 2-D local refinement in PLTMG; $\mathbf{t}_{i j}=$ $\left(\mathbf{t}_{i}+\mathbf{t}_{j}\right) / 2, i<j$. (a) Regular refinement; no interior angle is greater than $\pi / 2$. (b) Regular refinement; one interior angle is greater than $\pi / 2$. (c) Irregular refinement 


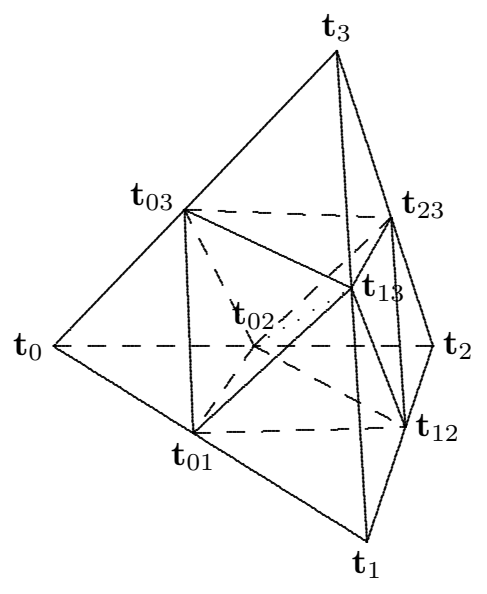

FiguRE 2. Illustration for the regular refinement (8subtetrahedron subdivision) $\mathrm{SUB}_{8} ; \mathbf{t}_{i j}=\left(\mathbf{t}_{i}+\mathbf{t}_{j}\right) / 2, i<j$; eight subtetrahedra are $\mathbf{T}_{1}^{1}\left(\mathbf{t}_{0}, \mathbf{t}_{01}, \mathbf{t}_{02}, \mathbf{t}_{03}\right), \mathbf{T}_{2}^{1}\left(\mathbf{t}_{01}, \mathbf{t}_{1}, \mathbf{t}_{12}, \mathbf{t}_{13}\right)$, $\mathbf{T}_{3}^{1}\left(\mathbf{t}_{02}, \mathbf{t}_{12}, \mathbf{t}_{2}, \mathbf{t}_{23}\right), \quad \mathbf{T}_{4}^{1}\left(\mathbf{t}_{03}, \mathbf{t}_{13}, \mathbf{t}_{23}, \mathbf{t}_{3}\right), \quad \mathbf{T}_{5}^{1}\left(\mathbf{t}_{01}, \mathbf{t}_{13}, \mathbf{t}_{03}, \mathbf{t}_{02}\right)$, $\mathbf{T}_{6}^{1}\left(\mathbf{t}_{01}, \mathbf{t}_{12}, \mathbf{t}_{13}, \mathbf{t}_{02}\right), \mathbf{T}_{7}^{1}\left(\mathbf{t}_{23}, \mathbf{t}_{02}, \mathbf{t}_{12}, \mathbf{t}_{13}\right)$, and $\mathbf{T}_{8}^{1}\left(\mathbf{t}_{23}, \mathbf{t}_{03}, \mathbf{t}_{02}, \mathbf{t}_{13}\right)$

Given a tetrahedron $\mathbf{T}, \mathrm{SUB}_{8}$ can be performed to $\mathbf{T}$ and its subtetrahedra repeatedly to produce a sequence of meshes. Note that the center edge in $\mathrm{SUB}_{8}$ can be any one of the three choices (e.g., $\mathbf{t}_{01} \mathbf{t}_{23}, \mathbf{t}_{02} \mathbf{t}_{13}$, or $\mathbf{t}_{03} \mathbf{t}_{12}$ ). Different strategies for choosing center edges will produce substantially different meshes in terms of the shape of tetrahedra in refined meshes. In $\S 2$, we describe a refinement procedure, i.e., a way of selecting the center edges, such that (a) iteratively applying $\mathrm{SUB}_{8}$ to any tetrahedron $\mathbf{T}$ produces at most three classes of similar tetrahedra, and (b) $0.5 \eta(\mathbf{T}) \leq \eta\left(\mathbf{T}_{i}^{n}\right) \leq 2 \eta(\mathbf{T})$, where $\mathbf{T}_{i}^{n}$ is any refined tetrahedron of $\mathbf{T}$ and $\eta$ is the mean ratio [10], a tetrahedron shape measure. In $\S 3$, we present an LRA extensively based on $\mathrm{SUB}_{8}$, and some properties of the algorithm are established. In $\S 4$, experimental results are provided.

\section{Subdivision OF A SINGLE TETRAHEDRON}

In this section, we are mainly concerned about the quality or the shape of tetrahedra of meshes produced by repeatedly performing $\mathrm{SUB}_{8}$ to a single tetrahedron $\mathbf{T}$ without considering the conformity of the refined meshes (which will be discussed in the next section). We first give some notation. For any (nondegenerate) tetrahedron $\mathbf{T}\left(\mathbf{t}_{0}, \mathbf{t}_{1}, \mathbf{t}_{2}, \mathbf{t}_{3}\right)$ with $\mathbf{t}_{0}, \mathbf{t}_{1}, \mathbf{t}_{2}, \mathbf{t}_{3}$ as the coordinates of the four vertices in the form of column vectors in $E^{3}$, define the $3 \times 3$ nonsingular matrix $T=\left[\mathbf{t}_{1}-\mathbf{t}_{0}, \mathbf{t}_{2}-\mathbf{t}_{0}, \mathbf{t}_{3}-\mathbf{t}_{0}\right]$. Note that the matrix has the same name as the tetrahedron but italic font is used instead of bold font, and $T$ depends on the ordering of vertices of $\mathbf{T}$. For any two tetrahedra $\mathbf{S}\left(\mathbf{s}_{0}, \mathbf{s}_{1}, \mathbf{s}_{2}, \mathbf{s}_{3}\right)$ and $\mathbf{T}\left(\mathbf{t}_{0}, \mathbf{t}_{1}, \mathbf{t}_{2}, \mathbf{t}_{3}\right)$, define the $3 \times 3$ matrices $M(\mathbf{S}, \mathbf{T})=T S^{-1}$ and $A(\mathbf{S}, \mathbf{T})=M^{T}(\mathbf{S}, \mathbf{T}) M(\mathbf{S}, \mathbf{T})$. Note that $M$ and $A$ depend on the ordering of tetrahedron vertices, and $M$ is the matrix defining the affine transformation from $\mathbf{S}$ to $\mathbf{T}$ such that $\mathbf{t}_{i}=M(\mathbf{S}, \mathbf{T}) \mathbf{s}_{i}+\mathbf{b}, 0 \leq i \leq 3$, where $\mathbf{b}=\mathbf{t}_{0}-M(\mathbf{S}, \mathbf{T}) \mathbf{s}_{0}$. 
The tetrahedron shape measure $\eta$, defined in [10], is used in analyzing the quality of meshes generated by our algorithm. The mean ratio $\eta$ approaches zero for poorlyshaped tetrahedra, and attains its maximum value 1 for the regular tetrahedron. In [11], we proved that $\eta$ is "equivalent" to two commonly used shape measures, minimum solid angle $\theta_{\min }$ and radius ratio $\rho$, in the sense that if $\eta$ approaches zero or reaches its maximum value, so do $\theta_{\min }$ and $\rho$. Other properties of $\eta$ and the relationship between $\eta$ and other shape measures can be found in $[10,11]$. The following two formulae are given in [10], and will be often used in the remainder of this paper:

$$
\eta(\mathbf{T})=3 \sqrt[3]{\lambda_{1} \lambda_{2} \lambda_{3}} /\left(\lambda_{1}+\lambda_{2}+\lambda_{3}\right)=3 \sqrt[3]{\operatorname{det}(A(\mathbf{R}, \mathbf{T}))} / \operatorname{trace}(A(\mathbf{R}, \mathbf{T})),
$$

where $\lambda_{1}, \lambda_{2}$, and $\lambda_{3}$ are the eigenvalues of the matrix $A(\mathbf{R}, \mathbf{T}), \mathbf{R}$ is a regular tetrahedron with the same volume as $\mathbf{T}$, and $\eta$ is independent of the ordering of tetrahedron vertices and of the vertex coordinates of $\mathbf{R}$, and

$$
\eta(\mathbf{T})=12(3 v)^{2 / 3} / \sum_{0 \leq i<j \leq 3} l_{i j}^{2},
$$

where $v$ is the volume of $\mathbf{T}$ and the $l_{i j}$ are the lengths of the edges of $\mathbf{T}$.

As mentioned in the previous section, the center edge in $\mathrm{SUB}_{8}$ is the edge connecting a pair of opposite edges, called the base edges. That is, the center edge is the edge joining the midpoints of a pair of base edges. We say that a subtetrahedron has the same subdivision pattern as its direct parent if its base edges include either the center edge or a half of a base edge of its parent, e.g., in Figure 2, if the base edges of $\mathbf{T}_{1}^{1}\left(\mathbf{t}_{0}, \mathbf{t}_{01}, \mathbf{t}_{02}, \mathbf{t}_{03}\right)$ are $\mathbf{t}_{0} \mathbf{t}_{02}$ and $\mathbf{t}_{01} \mathbf{t}_{03}$, then $\mathbf{T}_{1}^{1}\left(\mathbf{t}_{0}, \mathbf{t}_{01}, \mathbf{t}_{02}, \mathbf{t}_{03}\right)$ has the same subdivision pattern as $\mathbf{T}\left(\mathbf{t}_{0}, \mathbf{t}_{1}, \mathbf{t}_{2}, \mathbf{t}_{3}\right)$, since $\mathbf{t}_{0} \mathbf{t}_{02}$ is a half of the base edge $\mathbf{t}_{0} \mathbf{t}_{2}$ of $\mathbf{T}$. It can be easily verified that if a subtetrahedron has the same subdivision pattern as its parent, then its two base edges and center edge are uniquely determined. Now we describe a procedure SUBDIV for iteratively applying SUB $_{8}$ to $\mathbf{T}$.

\section{Algorithm SUBDIV.}

(1) Subdivide $\mathbf{T}=\mathbf{T}_{1}^{0}$ into eight subtetrahedra using $\mathrm{SUB}_{8}$ (see Figure 2); the center edge can be any of the three choices, i.e., $\mathbf{t}_{01} \mathbf{t}_{23}, \mathbf{t}_{02} \mathbf{t}_{13}$, or $\mathbf{t}_{03} \mathbf{t}_{12}$; label the eight subtetrahedra by $\mathbf{T}_{i}^{1}, 1 \leq i \leq 8$, and let $\mathbf{T}_{i}^{1}$ have the same subdivision pattern as $\mathbf{T} ; n:=1$.

(2) Subdivide $\mathbf{T}_{i}^{n}, 1 \leq i \leq 8^{n}$, using $\mathrm{SUB}_{8}$, and let its subtetrahedra have the same subdivision pattern as $\mathbf{T}_{i}^{n}$; label the resulting subtetrahedra by $\mathbf{T}_{i}^{n+1}$, $1 \leq i \leq 8^{n+1}$.

(3) $n:=n+1$; repeat (2) or terminate the subdivision.

The superscript $n$ in $\mathbf{T}_{i}^{n}$ denotes the level of subdivision. We assume that each subtetrahedron is subdivided to the same level (this constraint will be removed in the next section). In order to study the quality of the mesh produced by SUBDIV (which is a main procedure in our LRA in the next section), we first describe SUBDIV by another equivalent procedure, which is relatively easy to use in analyzing the shape of the refined meshes. To this end, we introduce a canonical tetrahedron $\mathbf{P}$ called a rhombic tetrahedron [15], shown in Figure 3, which is used in an alternative description of SUBDIV. $\mathbf{P}$ has two longer edges of the same length and four 


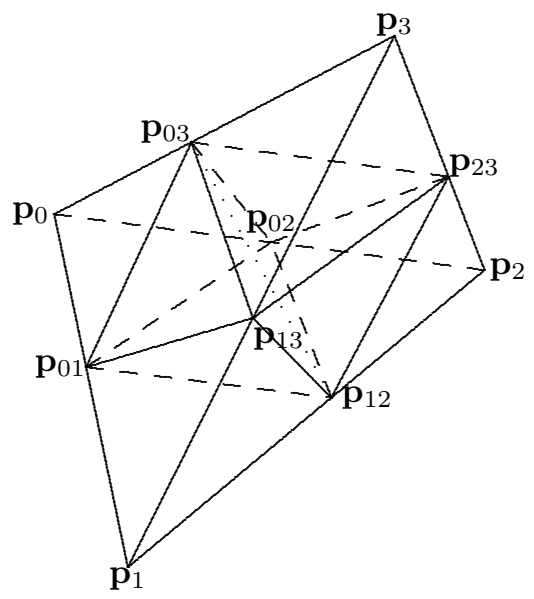

Figure 3. Rhombic tetrahedron $\mathbf{P}\left(\mathbf{p}_{\mathbf{0}}, \mathbf{p}_{1}, \mathbf{p}_{2}, \mathbf{p}_{3}\right)$ with $\left|\mathbf{p}_{0} \mathbf{p}_{1}\right|=$ $\left|\mathbf{p}_{2} \mathbf{p}_{3}\right|=\left|\mathbf{p}_{1} \mathbf{p}_{3}\right|=\left|\mathbf{p}_{0} \mathbf{p}_{2}\right|=m,\left|\mathbf{p}_{0} \mathbf{p}_{3}\right|=\left|\mathbf{p}_{1} \mathbf{p}_{2}\right|=2 \mathrm{~m} / \sqrt{3}$ $\mathbf{p}_{i j}=\left(\mathbf{p}_{i}+\mathbf{p}_{j}\right) / 2$. The coordinates are $\mathbf{p}_{0}(-\sqrt{2} m / \sqrt{3}, 0,0)$, $\mathbf{p}_{1}(0,-m / \sqrt{3}, 0), \mathbf{p}_{2}(0, m / \sqrt{3}, 0), \mathbf{p}_{3}(0,0, \sqrt{2} m / \sqrt{3}), \mathbf{p}_{01}(-\sqrt{6} m / 6$, $-\sqrt{3} m / 6,0), \mathbf{p}_{02}(-\sqrt{6} m / 6, \sqrt{3} m / 6,0), \mathbf{p}_{03}(-\sqrt{6} m / 6,0, \sqrt{6} m / 6)$, $\mathbf{p}_{12}(0,0,0), \mathbf{p}_{13}(0,-\sqrt{3} m / 6, \sqrt{6} m / 6)$, and $\mathbf{p}_{23}(0, \sqrt{3} m / 6, \sqrt{6} m / 6)$

shorter edges of the same length. The most important property of $\mathbf{P}$ is given in the following lemma, which is proved in [15].

Lemma 1. If $S U B_{8}$ is applied to $\mathbf{P}$ with the center edge connecting its longest edges $\mathbf{p}_{0} \mathbf{p}_{3}$ and $\mathbf{p}_{1} \mathbf{p}_{2}$, then the eight subtetrahedra $\mathbf{P}_{1}^{1}\left(\mathbf{p}_{0}, \mathbf{p}_{01}, \mathbf{p}_{02}, \mathbf{p}_{03}\right)$, $\mathbf{P}_{2}^{1}\left(\mathbf{p}_{01}, \mathbf{p}_{1}, \mathbf{p}_{12}, \mathbf{p}_{13}\right), \quad \mathbf{P}_{3}^{1}\left(\mathbf{p}_{02}, \mathbf{p}_{12}, \mathbf{p}_{2}, \mathbf{p}_{23}\right), \quad \mathbf{P}_{4}^{1}\left(\mathbf{p}_{03}, \mathbf{p}_{13}, \mathbf{p}_{23}, \mathbf{p}_{3}\right), \quad \mathbf{P}_{5}^{1}\left(\mathbf{p}_{01}, \mathbf{p}_{12}\right.$, $\left.\mathbf{p}_{03}, \mathbf{p}_{13}\right), \mathbf{P}_{6}^{1}\left(\mathbf{p}_{23}, \mathbf{p}_{03}, \mathbf{p}_{12}, \mathbf{p}_{13}\right), \mathbf{P}_{7}^{1}\left(\mathbf{p}_{01}, \mathbf{p}_{03}, \mathbf{p}_{12}, \mathbf{p}_{02}\right)$, and $\mathbf{P}_{8}^{1}\left(\mathbf{p}_{23}, \mathbf{p}_{12}, \mathbf{p}_{03}, \mathbf{p}_{02}\right)$ are all similar to the original tetrahedron $\mathbf{P}\left(\mathbf{p}_{0}, \mathbf{p}_{1}, \mathbf{p}_{2}, \mathbf{p}_{3}\right)$ (see Figure 3$)$.

When $\mathrm{SUB}_{8}$ is applied to $\mathbf{P}$, the base edges of $\mathbf{P}$ are its longest edges $\left(\mathbf{p}_{0} \mathbf{p}_{3}\right.$, $\mathbf{p}_{1} \mathbf{p}_{2}$ ). With each subtetrahedron having the same subdivision pattern as its parent, it is obvious that the base edges of $\mathbf{P}_{i}^{1}, 1 \leq i \leq 4$, are its longest edges. Since $\left|\mathbf{p}_{03} \mathbf{p}_{12}\right|=\left|\mathbf{p}_{0} \mathbf{p}_{3}\right| / 2=\left|\mathbf{p}_{1} \mathbf{p}_{2}\right| / 2$, the base edges of $\mathbf{P}_{i}^{1}, 5 \leq i \leq 8$, are also its longest edges. Therefore, by Lemma 1 and induction, the base edges of any refined subtetrahedron of $\mathbf{P}$ produced by $\mathrm{SUB}_{8}$ are always its two longest edges.

Let $\mathbf{T}$ be any tetrahedron, and $\mathbf{P}$ be the canonical tetrahedron in Figure 3 . We describe another procedure TRANSUB which uses an affine transformation, and prove that SUBDIV and TRANSUB are equivalent.

\section{Algorithm TRANSUB.}

(1) Transform $\mathbf{T}$ to $\mathbf{P}$ by an affine transformation $\mathbf{y}=M^{-1}(\mathbf{P}, \mathbf{T}) \mathbf{x}+\mathbf{b}_{0}$, with the constraint that the two base edges of $\mathbf{T}$ in step (1) of SUBDIV are transformed into the pair of edges with the longest length in $\mathbf{P}$, i.e., $\mathbf{p}_{0} \mathbf{p}_{3}$ and $\mathbf{p}_{1} \mathbf{p}_{2}$ in Figure 3.

(2) Iteratively subdivide $\mathbf{P}$ to some level, with each subtetrahedron having the same subdivision pattern as its parent (i.e., the two base edges are the two longest edges). 
(3) Transform all subtetrahedra in $\mathbf{P}$ back to subtetrahedra in $\mathbf{T}$ using the inverse affine transformation $\mathbf{y}=M(\mathbf{P}, \mathbf{T}) \mathbf{x}+\mathbf{b}_{1}$.

Theorem 1. For any tetrahedron $\mathbf{T}$, if each subtetrahedron is refined to the same level, SUBDIV and TRANSUB produce the same set of refined tetrahedra.

Proof. The theorem can be established by using the fact that the midpoint of a line segment is transformed into the midpoint of the corresponding line segment under an affine transformation.

We now use TRANSUB to establish the main properties of meshes produced by SUBDIV.

Theorem 2. There are at most three classes of similar tetrahedra produced by SUBDIV in all the refined subtetrahedra of $\mathbf{T}$.

Proof. We define two tetrahedra to be similar to each other if one can be transformed into the other by translation, rotation, reflection, and uniform scaling (i.e., the scale factors for the three coordinate axes are the same). Let $\mathbf{T}\left(\mathbf{t}_{0}, \mathbf{t}_{1}, \mathbf{t}_{2}, \mathbf{t}_{3}\right)$ be a tetrahedron with vertices $\mathbf{t}_{0}, \mathbf{t}_{1}, \mathbf{t}_{2}, \mathbf{t}_{3}$. Define

$$
\mathcal{F}\left(\mathbf{T}\left(\mathbf{t}_{0}, \mathbf{t}_{1}, \mathbf{t}_{2}, \mathbf{t}_{3}\right), k, \mathbf{b}\right)=\mathbf{T}^{\prime}\left(k \mathbf{t}_{0}+\mathbf{b}, k \mathbf{t}_{1}+\mathbf{b}, k \mathbf{t}_{2}+\mathbf{b}, k \mathbf{t}_{3}+\mathbf{b}\right),
$$

where $k$ is a nonzero constant and $\mathbf{b}$ is a vector in $E^{3}$. Then $\mathbf{T}$ is similar to $\mathcal{F}(\mathbf{T}, k, \mathbf{b})$.

Let $\mathbf{T}$ be the tetrahedron shown in Figure 2, and $\mathbf{t}_{02} \mathbf{t}_{13}$ be the center edge. Let the eight subtetrahedra be labeled $\mathbf{T}_{1}^{1}\left(\mathbf{t}_{0}, \mathbf{t}_{01}, \mathbf{t}_{02}, \mathbf{t}_{03}\right), \quad \mathbf{T}_{2}^{1}\left(\mathbf{t}_{01}, \mathbf{t}_{1}, \mathbf{t}_{12}, \mathbf{t}_{13}\right)$, $\mathbf{T}_{3}^{1}\left(\mathbf{t}_{02}, \mathbf{t}_{12}, \mathbf{t}_{2}, \mathbf{t}_{23}\right), \mathbf{T}_{4}^{1}\left(\mathbf{t}_{03}, \mathbf{t}_{13}, \mathbf{t}_{23}, \mathbf{t}_{3}\right), \mathbf{T}_{5}^{1}\left(\mathbf{t}_{01}, \mathbf{t}_{13}, \mathbf{t}_{03}, \mathbf{t}_{02}\right), \mathbf{T}_{6}^{1}\left(\mathbf{t}_{01}, \mathbf{t}_{12}, \mathbf{t}_{13}, \mathbf{t}_{02}\right)$, $\mathbf{T}_{7}^{1}\left(\mathbf{t}_{23}, \mathbf{t}_{02}, \mathbf{t}_{12}, \mathbf{t}_{13}\right)$, and $\mathbf{T}_{8}^{1}\left(\mathbf{t}_{23}, \mathbf{t}_{03}, \mathbf{t}_{02}, \mathbf{t}_{13}\right)$. We shall prove that all subtetrahedra produced by SUBDIV are similar to one of $\mathbf{T}, \mathbf{T}_{5}^{1}$, and $\mathbf{T}_{6}^{1}$.

After one level of subdivision, the subtetrahedra $\mathbf{T}_{i}^{1}, 1 \leq i \leq 4$, at the four corners are similar to $\mathbf{T}$. Among the four interior subtetrahedra, $\mathbf{T}_{7}^{1}$ and $\mathbf{T}_{8}^{1}$ are similar to $\mathbf{T}_{5}^{1}$ and $\mathbf{T}_{6}^{1}$, respectively, since $\mathcal{F}\left(\mathbf{T}_{7}^{1}\left(\mathbf{t}_{23}, \mathbf{t}_{02}, \mathbf{t}_{12}, \mathbf{t}_{13}\right),-1, \mathbf{t}_{01}+\mathbf{t}_{23}\right)=$ $\mathbf{T}_{5}^{1}\left(\mathbf{t}_{01}, \mathbf{t}_{13}, \mathbf{t}_{03}, \mathbf{t}_{02}\right)$ and $\mathcal{F}\left(\mathbf{T}_{8}^{1}\left(\mathbf{t}_{23}, \mathbf{t}_{03}, \mathbf{t}_{02}, \mathbf{t}_{13}\right),-1, \mathbf{t}_{01}+\mathbf{t}_{23}\right)=\mathbf{T}_{6}^{1}\left(\mathbf{t}_{01}, \mathbf{t}_{12}, \mathbf{t}_{13}, \mathbf{t}_{02}\right)$.

After two levels of subdivision, we need to consider only subtetrahedra of $\mathbf{T}_{5}^{1}$ and $\mathbf{T}_{6}^{1}$. For the subdivision of $\mathbf{T}_{5}^{1}$ (or $\mathbf{T}_{6}^{1}$ ), we can relabel the vertex indices of Figure 2 as $0 \rightarrow 01,1 \rightarrow 02,2 \rightarrow 03,3 \rightarrow 13$ (or $0 \rightarrow 01,1 \rightarrow 02$, $2 \rightarrow 12,3 \rightarrow 13)$. By the above discussion, it suffices to prove that $\mathbf{T}_{55}^{2}\left(\mathbf{t}_{0213}, \mathbf{t}_{0103}, \mathbf{t}_{0102}, \mathbf{t}_{0113}\right), \quad \mathbf{T}_{56}^{2}\left(\mathbf{t}_{0213}, \mathbf{t}_{0203}, \mathbf{t}_{0103}, \mathbf{t}_{0102}\right), \quad \mathbf{T}_{65}^{2}\left(\mathbf{t}_{0213}, \mathbf{t}_{0102}, \mathbf{t}_{0112}\right.$, $\left.\mathbf{t}_{0113}\right)$, and $\mathbf{T}_{66}^{2}\left(\mathbf{t}_{0102}, \mathbf{t}_{0112}, \mathbf{t}_{0212}, \mathbf{t}_{0213}\right)$ are similar to one of $\mathbf{T}, \mathbf{T}_{5}^{1}$, and $\mathbf{T}_{6}^{1}$, where $\mathbf{t}_{i_{0} i_{1} i_{2} i_{3}}=\left(\mathbf{t}_{i_{0} i_{1}}+\mathbf{t}_{i_{2} i_{3}}\right) / 2=\left(\mathbf{t}_{i_{0}}+\mathbf{t}_{i_{1}}+\mathbf{t}_{i_{2}}+\mathbf{t}_{i_{3}}\right) / 4,0 \leq i_{0}, i_{1}, i_{2}, i_{3} \leq 3$. It is straightforward to verify that

$$
\begin{aligned}
& \mathcal{F}\left(\mathbf{T}_{55}^{2}\left(\mathbf{t}_{0213}, \mathbf{t}_{0103}, \mathbf{t}_{0102}, \mathbf{t}_{0113}\right),-2, \mathbf{t}_{13}+2 \mathbf{t}_{0102}\right)=\mathbf{T}_{6}^{1}\left(\mathbf{t}_{01}, \mathbf{t}_{12}, \mathbf{t}_{13}, \mathbf{t}_{02}\right), \\
& \mathcal{F}\left(\mathbf{T}_{56}^{2}\left(\mathbf{t}_{0213}, \mathbf{t}_{0203}, \mathbf{t}_{0103}, \mathbf{t}_{0102}\right),-4, \mathbf{t}_{3}+4 \mathbf{t}_{0102}\right)=\mathbf{T}\left(\mathbf{t}_{0}, \mathbf{t}_{1}, \mathbf{t}_{2}, \mathbf{t}_{3}\right), \\
& \mathcal{F}\left(\mathbf{T}_{65}^{2}\left(\mathbf{t}_{0213}, \mathbf{t}_{0102}, \mathbf{t}_{0112}, \mathbf{t}_{0113}\right),-2, \mathbf{t}_{23}+2 \mathbf{t}_{01}\right)=\mathbf{T}_{5}^{1}\left(\mathbf{t}_{01}, \mathbf{t}_{13}, \mathbf{t}_{03}, \mathbf{t}_{02}\right), \\
& \mathcal{F}\left(\mathbf{T}_{66}^{2}\left(\mathbf{t}_{0102}, \mathbf{t}_{0112}, \mathbf{t}_{0212}, \mathbf{t}_{0213}\right), 4,-\mathbf{t}_{0}-\mathbf{t}_{1}-\mathbf{t}_{2}\right)=\mathbf{T}\left(\mathbf{t}_{0}, \mathbf{t}_{1}, \mathbf{t}_{2}, \mathbf{t}_{3}\right) .
\end{aligned}
$$

Thus, all subtetrahedra at the second level are similar to one of $\mathbf{T}, \mathbf{T}_{5}^{1}$, and $\mathbf{T}_{6}^{1}$.

Consequently, further levels of subdivision do not introduce any more classes of similar tetrahedra, so the total number of classes of similar tetrahedra in all refined subtetrahedra of $\mathbf{T}$ is finite and bounded above by 3 .

Theorem 2 implies that the shape of subtetrahedra does not deteriorate arbitrarily. Moreover, the following theorem shows that the shape of the subtetrahedra 
are at least half as good as the shape of the very first tetrahedron $\mathbf{T}$ in terms of the shape measure $\eta$.

Theorem 3. For any refined subtetrahedron $\mathbf{T}_{i}^{n}$ of $\mathbf{T}$, produced by SUBDIV,

$$
0.5 \eta(\mathbf{T}) \leq \eta\left(\mathbf{T}_{i}^{n}\right) \leq 2 \eta(\mathbf{T}),
$$

and the lower bound is tight.

Proof. Let $M(\mathbf{P}, \mathbf{T})$ and $M(\mathbf{R}, \mathbf{P})$ be the two matrices defining the affine transformations from $\mathbf{P}$ to $\mathbf{T}$ and $\mathbf{R}$ to $\mathbf{P}$, respectively. Without loss of generality, we assume that $\mathbf{T}, \mathbf{P}$, and $\mathbf{R}$ have the same volume. Using the notation given at the beginning of this section, we have

$$
T=M(\mathbf{P}, \mathbf{T}) P=M(\mathbf{P}, \mathbf{T}) M(\mathbf{R}, \mathbf{P}) R,
$$

where $T, P$ and $R$ are the matrices derived from $\mathbf{T}, \mathbf{P}$ and $\mathbf{R}$. From step (3) of TRANSUB, the tetrahedron $\mathbf{T}_{i}^{n}$ is transformed from $\mathbf{P}_{i}^{n}$ using $M(\mathbf{P}, \mathbf{T})$, so

$$
T_{i}^{n}=M(\mathbf{P}, \mathbf{T}) P_{i}^{n} .
$$

With a suitable ordering of vertices of $\mathbf{P}_{i}^{n}$, one has $P_{i}^{n}=\alpha Q P$, where $\alpha$ is a positive constant and $Q$ is an orthogonal matrix, since $\mathbf{P}_{i}^{n}$ is similar to $\mathbf{P}$ by Lemma 1 . So

$$
\begin{aligned}
T_{i}^{n} & =\alpha M(\mathbf{P}, \mathbf{T}) Q P=\alpha M(\mathbf{P}, \mathbf{T}) Q M(\mathbf{R}, \mathbf{P}) R \\
& =M(\mathbf{P}, \mathbf{T}) Q M(\mathbf{R}, \mathbf{P}) R^{n}=M\left(\mathbf{R}^{n}, \mathbf{T}_{i}^{n}\right) R^{n},
\end{aligned}
$$

where $\mathbf{R}^{n}$ is a regular tetrahedron having the same volume as $\mathbf{T}_{i}^{n}$.

From $\operatorname{det}(A(\mathbf{R}, \mathbf{T}))=\operatorname{det}\left(A\left(\mathbf{R}^{n}, \mathbf{T}_{i}^{n}\right)\right)=1$ and (1), we have

$$
\begin{aligned}
\eta(\mathbf{T}) & =\frac{3 \sqrt[3]{\operatorname{det}(A(\mathbf{R}, \mathbf{T}))}}{\operatorname{trace}(A(\mathbf{R}, \mathbf{T}))} \\
& =\frac{3}{\operatorname{trace}\left((M(\mathbf{P}, \mathbf{T}) M(\mathbf{R}, \mathbf{P}))^{T} M(\mathbf{P}, \mathbf{T}) M(\mathbf{R}, \mathbf{P})\right)}, \\
\eta\left(\mathbf{T}_{i}^{n}\right) & =\frac{3 \sqrt{\operatorname{det}\left(A\left(\mathbf{R}^{n}, \mathbf{T}_{i}^{n}\right)\right)}}{\operatorname{trace}\left(A\left(\mathbf{R}^{n}, \mathbf{T}_{i}^{n}\right)\right)} \\
& =\frac{3}{\operatorname{trace}\left((M(\mathbf{P}, \mathbf{T}) Q M(\mathbf{R}, \mathbf{P}))^{T} M(\mathbf{P}, \mathbf{T}) Q M(\mathbf{R}, \mathbf{P})\right)} .
\end{aligned}
$$

Let $\|B\|_{F}$ denote the Frobenius norm of the matrix $B$ [3], i.e.,

$$
\|B\|_{F}=\left(\operatorname{trace}\left(B^{T} B\right)\right)^{1 / 2} .
$$

By (4) and (5), it follows that

$$
\frac{\eta\left(\mathbf{T}_{i}^{n}\right)}{\eta(\mathbf{T})}=\frac{\|M(\mathbf{P}, \mathbf{T}) M(\mathbf{R}, \mathbf{P})\|_{F}^{2}}{\|M(\mathbf{P}, \mathbf{T}) Q M(\mathbf{R}, \mathbf{P})\|_{F}^{2}} .
$$

By the singular value decomposition [3], $M(\mathbf{P}, \mathbf{T})=Q_{1} \operatorname{diag}\left(\sqrt{\lambda_{1}}, \sqrt{\lambda_{2}}, \sqrt{\lambda_{3}}\right) Q_{2}$, where $Q_{1}, Q_{2}$ are orthogonal matrices and $\lambda_{1}, \lambda_{2}, \lambda_{3}$ are the eigenvalues of $A(\mathbf{P}, \mathbf{T})$ $\left(\sqrt{\lambda_{1}}, \sqrt{\lambda_{2}}, \sqrt{\lambda_{3}}\right.$ are the singular values of $\left.M(\mathbf{P}, \mathbf{T})\right)$. It follows that

$$
\begin{gathered}
\|M(\mathbf{P}, \mathbf{T}) M(\mathbf{R}, \mathbf{P})\|_{F}^{2}=\left\|\operatorname{diag}\left(\sqrt{\lambda_{1}}, \sqrt{\lambda_{2}}, \sqrt{\lambda_{3}}\right) Q_{2} M(\mathbf{R}, \mathbf{P})\right\|_{F}^{2}, \\
\|M(\mathbf{P}, \mathbf{T}) Q M(\mathbf{R}, \mathbf{P})\|_{F}^{2}=\left\|\operatorname{diag}\left(\sqrt{\lambda_{1}}, \sqrt{\lambda_{2}}, \sqrt{\lambda_{3}}\right) Q_{2} Q M(\mathbf{R}, \mathbf{P})\right\|_{F}^{2} .
\end{gathered}
$$

Let $\mathbf{R}\left(\mathbf{r}_{0}, \mathbf{r}_{1}, \mathbf{r}_{2}, \mathbf{r}_{3}\right)$ be a regular tetrahedron having the same volume as $\mathbf{P}$ with coordinates $\mathbf{r}_{0}=(-\sqrt{3} a / 2,0,0)^{T}, \mathbf{r}_{1}=(0,-a / 2,0)^{T}, \mathbf{r}_{2}=(0, a / 2,0)^{T}$, and $\mathbf{r}_{3}=$ 
$(-\sqrt{3} a / 6,0, \sqrt{6} a / 3)^{T}$. From the coordinates shown in Figure 3 , with $\mathbf{r}_{i}$ being transformed to $\mathbf{p}_{i}$, we have

$$
M(\mathbf{R}, \mathbf{P})=k\left[\begin{array}{ccc}
2 \sqrt{2} & 0 & 1 \\
0 & 2 \sqrt{3} & 0 \\
0 & 0 & 3
\end{array}\right],
$$

where $k^{3}=\sqrt{6} / 72$. If $U$ is an orthogonal matrix with elements $u_{i j}, 1 \leq i, j \leq 3$, we have

$$
\begin{aligned}
\left\|\operatorname{diag}\left(\sqrt{\lambda_{1}}, \sqrt{\lambda_{2}}, \sqrt{\lambda_{3}}\right) U M(\mathbf{R}, \mathbf{P})\right\|_{F}^{2} & \\
=k^{2}\left(\lambda_{1}\left[9+3\left(1-\left(u_{11}-u_{13}\right)^{2}\right)\right]+\right. & \lambda_{2}\left[9+3\left(1-\left(u_{21}-u_{23}\right)^{2}\right)\right] \\
& \left.\quad+\lambda_{3}\left[9+3\left(1-\left(u_{31}-u_{33}\right)^{2}\right)\right]\right) .
\end{aligned}
$$

Since $0 \leq(x+y)^{2} \leq 2$ for any $x$, $y$ satisfying $x^{2}+y^{2} \leq 1$, we have

$$
6 k^{2}\left(\lambda_{1}+\lambda_{2}+\lambda_{3}\right) \leq\left\|\operatorname{diag}\left(\sqrt{\lambda_{1}}, \sqrt{\lambda_{2}}, \sqrt{\lambda_{3}}\right) U M(\mathbf{R}, \mathbf{P})\right\|_{F}^{2} \leq 12 k^{2}\left(\lambda_{1}+\lambda_{2}+\lambda_{3}\right) .
$$

Then combining (6), (7), and (8) yields

$$
0.5 \leq \frac{\eta\left(\mathbf{T}_{i}^{n}\right)}{\eta(\mathbf{T})} \leq 2 .
$$

We now prove that the lower bound cannot be improved. For any $\epsilon>0$, we define a tetrahedron $\mathbf{T}$ with vertices $\mathbf{t}_{0}=\left(0, \frac{\sqrt{11(0.5-\epsilon)}}{\sqrt{8 \epsilon}}, 0\right), \mathbf{t}_{1}=(-1,0,0), \mathbf{t}_{2}=(1,0,0)$, and $\mathbf{t}_{3}=\left(0, \frac{\sqrt{11(0.5-\epsilon)}}{\sqrt{8 \epsilon}}, 1\right)$. Consider the subtetrahedron $\mathbf{T}_{1}^{1}=\left(\mathbf{t}_{03}, \mathbf{t}_{13}, \mathbf{t}_{12}, \mathbf{t}_{23}\right)$, where $\mathbf{t}_{i j}$ is the midpoint of $\mathbf{t}_{i}$ and $\mathbf{t}_{j}$. By straightforward computation using (2), we find $\eta\left(\mathbf{T}_{1}^{1}\right) / \eta(\mathbf{T})=0.5+\epsilon$. Hence the lower bound is tight.

In the subdivision procedure SUBDIV, the center edge added in the interior of a tetrahedron is selected by a specific rule. Otherwise, refined subtetrahedra with good shape may not be guaranteed. In fact, there is a means of selecting center edges such that the shape of some subtetrahedra become arbitrarily poor, as described by the following lemma (which is proved in [9]) and theorem.

Lemma 2. Suppose $S U B_{8}$ is applied to any tetrahedron $\mathbf{T}\left(\mathbf{t}_{0}, \mathbf{t}_{1}, \mathbf{t}_{2}, \mathbf{t}_{3}\right)$. If the center edge of $\mathbf{T}$ is chosen such that the minimum $\eta$-value of the four interior subtetrahedra achieves a minimum value among the three possible choices of center edges, then there exists a subtetrahedron $\mathbf{T}_{1}^{1}$ of $\mathbf{T}$ such that $\eta\left(\mathbf{T}_{1}^{1}\right) \leq 6 / 7 \eta(\mathbf{T})$, and the upper bound is tight.

Theorem 4. There exists a means of selecting the center edges such that after $n$ levels of refinement by applying $S U B_{8}$ to $\mathbf{T}$,

$$
\eta\left(\mathbf{T}_{i}^{n}\right) \leq(6 / 7)^{n} \eta(\mathbf{T}),
$$

for some refined subtetrahedron $\mathbf{T}_{i}^{n}$.

Proof. At each step of refinement, if the center edge is chosen as described in Lemma 2, the theorem can be established by repeatedly using Lemma 2.

In light of the scheme of selecting the center edge in Lemma 2, we can always choose the center edge such that the minimum shape measure of the four interior subtetrahedra achieves a maximum value, but it seems hard to obtain a finite number of classes of similar tetrahedra as in Theorem 2 and constant bounds as in Theorem 3. 


\section{LOCAL REFINEMENT OF A CONFORMING MESH}

Similar to the 2-D approach in [1, p. 26], we design a 3-D LRA mainly depending on the regular subdivision $\mathrm{SUB}_{8}$ illustrated in Figure 2, since the shape of refined tetrahedra produced by repeatedly performing $\mathrm{SUB}_{8}$ to a tetrahedron, by the discussion in the last section, are bounded below if each subtetrahedron has the same subdivision pattern as its parent. Simply applying $\mathrm{SUB}_{8}$ to a set of subtetrahedra of a conforming mesh may produce nonconformity between tetrahedra. Therefore, at the boundary of a refined region, other nonregular refinements may be needed to ensure a conforming mesh.

A split point is defined to be the midpoint of an edge whenever the edge needs subdivision. In an initial mesh $\mathcal{T}$, suppose a set $S$ of tetrahedra are chosen for refinement. A split point is added to each edge of each tetrahedron in $S$. Also, for any face of a tetrahedron not in $S$ that contains two split points, a split point is added to the edge that does not have a split point so that there are either one or three split points on each face. A face with 3 or 1 split point(s) is subdivided like Figure 1a or 1c, respectively; we do not use the subdivision of Figure 1b, since this will significantly increase the difficulty of keeping a conforming mesh. Under the above assumptions, the number of split points for a tetrahedron in the initial mesh can be 1, 2 (on a pair of opposite edges), 3 (on the same face), or 6 . According to the number of split points, the subdivision of a tetrahedron is illustrated in Figures $4 \mathrm{a}$, $4 \mathrm{~b}, 4 \mathrm{c}$, and Figure 2, respectively. The three nonregular refinements in Figures 4a, $4 \mathrm{~b}$, and $4 \mathrm{c}$ are denoted by $\mathrm{SUB}_{2}, \mathrm{SUB}_{4}^{1}$, and $\mathrm{SUB}_{4}^{2}$, respectively, and are applied to the boundary of a refined region. In the next refinement, if any tetrahedron, produced by $\mathrm{SUB}_{2}, \mathrm{SUB}_{4}^{1}$, or $\mathrm{SUB}_{4}^{2}$, is chosen for refinement, its parent is always refined by $\mathrm{SUB}_{8}$ first. Thus we never need to add a split point on an edge, called an irregular edge, which is generated by connecting a vertex to the midpoint of an edge of a face in $\mathrm{SUB}_{2}, \mathrm{SUB}_{4}^{1}$, or $\mathrm{SUB}_{4}^{2}$, e.g., $\mathbf{t}_{3} \mathbf{t}_{12}$ in Figure $4 \mathrm{a}$ and $\mathbf{t}_{03} \mathbf{t}_{12}$ in Figure 4b. An irregular face is defined to be one containing an irregular edge, e.g., face $\mathbf{t}_{1} \mathbf{t}_{3} \mathbf{t}_{12}$ in Figure 4a, and may have 0, 1, or 2 split points (for the next refinement). A face that is not irregular is called a regular face, and may have 0 , 1 , or 3 split points.

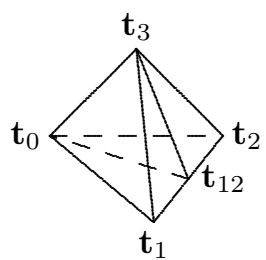

(a) $\mathrm{SUB}_{2}$

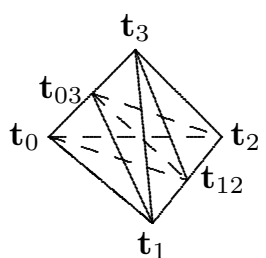

(b) $\mathrm{SUB}_{4}^{1}$

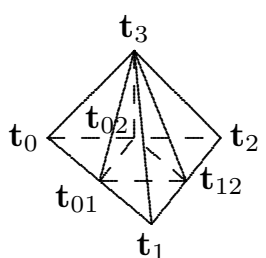

(c) $\mathrm{SUB}_{4}^{2}$

FIGURE 4. Illustration for nonregular refinements; $\mathbf{t}_{i j}=$ $\left(\mathbf{t}_{i}+\mathbf{t}_{j}\right) / 2, i<j$. (a) Subdivision of a tetrahedron with one split point $\left(\mathbf{t}_{12}\right)$. (b) Subdivision of a tetrahedron with two split points $\left(\mathbf{t}_{12}, \mathbf{t}_{03}\right)$ on a pair of opposite edges. (c) Subdivision of a tetrahedron with three split points $\left(\mathbf{t}_{01}, \mathbf{t}_{02}, \mathbf{t}_{12}\right)$ on the same face 
We now give a local refinement procedure, QLRS (quality local refinement based on subdivision), based on $\mathrm{SUB}_{8}, \mathrm{SUB}_{2}, \mathrm{SUB}_{4}^{1}$, and $\mathrm{SUB}_{4}^{2}$. A tetrahedron $\mathbf{T}$ is called a tetrahedron of type $S_{8}$ if $\mathbf{T}$ is either a tetrahedron in the original mesh, or produced by $\mathrm{SUB}_{8}$. Similarly, a tetrahedron of type $\mathrm{S}_{2}, \mathrm{~S}_{4}^{1}$, or $\mathrm{S}_{4}^{2}$ means that the tetrahedron is produced by $\mathrm{SUB}_{2}, \mathrm{SUB}_{4}^{1}$, or $\mathrm{SUB}_{4}^{2}$, respectively. In the following, we assume that whenever $\mathrm{SUB}_{8}$ is applied to a tetrahedron $\mathbf{T}$ of the original mesh $\mathcal{T}$, the center edge of $\mathbf{T}$ is chosen such that the minimum shape value of the four interior subtetrahedra (i.e., $\mathbf{T}_{5}^{1}, \mathbf{T}_{6}^{1}, \mathbf{T}_{7}^{1}, \mathbf{T}_{8}^{1}$ in Figure 2 ) of $\mathbf{T}$ achieves a maximum value in terms of the tetrahedron shape measure $\eta$. Using (2), finding such a center edge involves only comparisons among the squares of length of the edges of $\mathbf{T}$, since the subtetrahedra have identical volumes. For any tetrahedron $\mathbf{T}$, an unsplit edge of $\mathbf{T}$ is an edge that does not have a split point.

\section{Algorithm QLRS.}

(1) Set $\mathcal{T}^{0}:=\mathcal{T} ; m:=0$.

(2) Select a set $S_{m}$ of tetrahedra needing refinement from $\mathcal{T}^{m}$; for each tetrahedron $\mathbf{T}$ in $S_{m}$, if $\mathbf{T}$ is a tetrahedron of type $\mathrm{S}_{8}$, mark a split point on each unsplit edge of $\mathbf{T}$; otherwise, mark a split point on each unsplit edge of $\mathbf{T}$ 's parent.

(3) For each tetrahedron $\mathbf{T}$ of type $\mathrm{S}_{2}$ in $\mathcal{T}^{m}$ (see Figure 4a), whenever one of the edges $\mathbf{t}_{3} \mathbf{t}_{1}, \mathbf{t}_{3} \mathbf{t}_{2}$ ( or $\mathbf{t}_{0} \mathbf{t}_{1}, \mathbf{t}_{0} \mathbf{t}_{2}$ ) has a split point, mark a split point on the other if it does not have a split point; whenever $\mathbf{t}_{1} \mathbf{t}_{12}\left(\right.$ or $\left.\mathbf{t}_{2} \mathbf{t}_{12}\right)$ has a split point, mark a split point on each unsplit edge of T's parent. For each tetrahedron of type $\mathrm{S}_{4}^{1}$ or $\mathrm{S}_{4}^{2}$ in $\mathcal{T}^{m}$, whenever one of its regular edges has a split point, mark a split point on each unsplit edge of T's parent. In addition, whenever a regular face in $\mathcal{T}^{m}$ has two split points, mark a split point on the unsplit edge of the face; repeat the above process until no more split points are needed.

(4) For any $\mathbf{T}$ in $\mathcal{T}^{m}$ with a nonzero number of split points, if $\mathbf{T}$ is a tetrahedron of type $\mathrm{S}_{8}$, subdivide $\mathbf{T}$ using $\mathrm{SUB}_{8}, \mathrm{SUB}_{2}, \mathrm{SUB}_{4}^{1}$, or $\mathrm{SUB}_{4}^{2}$ according to the number of split points on $\mathbf{T}$, and let the subtetrahedra have the same subdivision pattern as $\mathbf{T}$; otherwise, remove T's sibling(s), subdivide T's parent $\mathbf{T}_{p}$ using $\mathrm{SUB}_{8}, \mathrm{SUB}_{4}^{1}$, or $\mathrm{SUB}_{4}^{2}$ according to the number of split points on $\mathbf{T}_{p}$, and subdivide each subtetrahedron of $\mathbf{T}_{p}$ if necessary using $\mathrm{SUB}_{2}$ or $\mathrm{SUB}_{4}^{2}$ according to the number of split points on the subtetrahedron; label the conforming mesh by $\mathcal{T}^{m+1}$.

(5) $m:=m+1$; go to (2) if necessary or terminate.

Note that superscript $m$ of $\mathcal{T}^{m}$ indicates that the mesh $\mathcal{T}^{m}$ is created after $m$ levels of refinement on $\mathcal{T}^{0}$. In step (3) of QLRS, whenever a split point is added to an edge of a tetrahedron $\mathbf{T}$ of type $\mathrm{S}_{4}^{1}$ or $\mathrm{S}_{4}^{2}$, the split points are also added to the edges of T's parent $\mathbf{T}_{p}$. This guarantees that a tetrahedron of type $\mathrm{S}_{4}^{1}$ or $\mathrm{S}_{4}^{2}$ will never be further refined, i.e., whenever a tetrahedron $\mathbf{T}$ of type $S_{4}^{1}$ or $S_{4}^{2}$ needs to be refined, $\mathbf{T}_{p}$ is always first refined by the regular refinement. For a tetrahedron $\mathbf{T}$ of type $\mathrm{S}_{2}$, its parent $\mathbf{T}_{p}$ may be subdivided by $\mathrm{SUB}_{4}^{1}, \mathrm{SUB}_{4}^{2}$, or $\mathrm{SUB}_{8}$, and no subdivision is needed for subtetrahedra of $\mathbf{T}_{p}$ if $\mathbf{T}_{p}$ is subdivided by $\mathrm{SUB}_{4}^{1}$ or $\mathrm{SUB}_{4}^{2}$. Figure 5 illustrates possible split points on subtetrahedra of $\mathbf{T}_{p}$ after $\mathrm{SUB}_{8}$ is applied to $\mathbf{T}_{p}$ in step (4) of QLRS.

It is obvious that step (3) of QLRS will terminate in a finite number of steps, since in the worst case each edge, which is not an irregular edge, of $\mathcal{T}^{m}$ has a split 


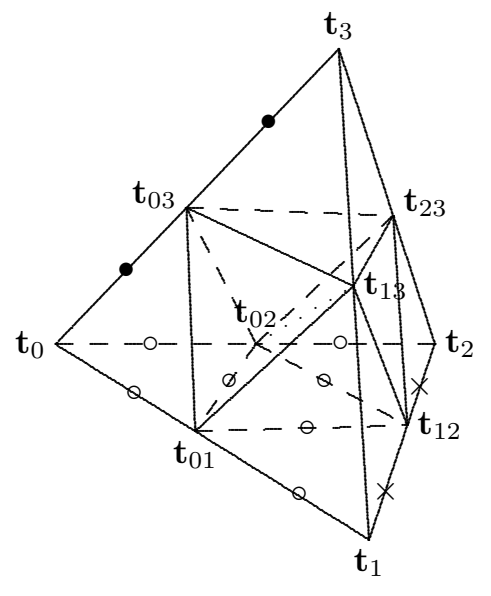

FigURE 5. Illustration for possible split points after $\mathrm{SUB}_{8}$ is applied to $\mathbf{T}$ 's parent $\mathbf{T}_{p}\left(\mathbf{t}_{0}, \mathbf{t}_{1}, \mathbf{t}_{2}, \mathbf{t}_{3}\right)$. Case 1: $\mathbf{T}$ is a tetrahedron of type $\mathrm{S}_{2}$ (see Figure $4 \mathrm{a}$ ); possible split points are on edges with $\times$. Case 2: $\mathbf{T}$ is a tetrahedron of type $\mathrm{S}_{4}^{1}$ (see Figure $4 \mathrm{~b}$ ); possible split points are on edges with $\times$ or $\bullet$. Case 3: $\mathbf{T}$ is a tetrahedron of type $S_{4}^{2}$ (see Figure 4c); possible split points are on edges with $\times$ or $\circ$

point. To guarantee the validity of QLRS, we need to prove that step (4) of QLRS produces a conforming mesh.

Theorem 5. If $\mathcal{T}^{m}$ is a conforming mesh, then step (4) of Algorithm QLRS produces a conforming mesh $\mathcal{T}^{m+1}$.

Proof. Let $\mathbf{T}$ be a tetrahedron of type $\mathrm{S}_{2}, \mathrm{~S}_{4}^{1}$, or $\mathrm{S}_{4}^{2}$ in $\mathcal{T}^{m}$, i.e., T's parent $\mathbf{T}_{p}$ has the configuration of Figure $4 \mathrm{a}, 4 \mathrm{~b}$, or $4 \mathrm{c}$ (we assume that $\mathbf{T}_{p}$ has tetrahedron vertices $\mathbf{t}_{0}, \mathbf{t}_{1}, \mathbf{t}_{2}$, and $\mathbf{t}_{3}$ ). We first prove that if $\mathrm{SUB}_{8}$ is applied to $\mathbf{T}_{p}$ in step (4) of QLRS, each face of a subtetrahedron of $\mathbf{T}_{p}$ has 0,1 , or 3 split points. Note that among edges of $\mathbf{T}$ or $\mathbf{T}$ 's sibling(s), only edges $\mathbf{t}_{1} \mathbf{t}_{12}, \mathbf{t}_{2} \mathbf{t}_{12}$ of Figure 4 a (see Case 1 in Figure 5); $\mathbf{t}_{0} \mathbf{t}_{03}, \mathbf{t}_{3} \mathbf{t}_{03}, \mathbf{t}_{1} \mathbf{t}_{12}, \mathbf{t}_{2} \mathbf{t}_{12}$ of Figure $4 \mathrm{~b}$ (see Case 2 in Figure 5 ); and all 9 edges on face $\mathbf{t}_{0} \mathbf{t}_{1} \mathbf{t}_{2}$ of Figure $4 \mathrm{c}$ (see Case 3 in Figure 5) exist in subtetrahedra produced by applying $\mathrm{SUB}_{8}$ to $\mathbf{T}_{p}$. It can be easily verified that each face of a subtetrahedron of $\mathbf{T}_{p}$ has 0 , 1 , or 3 edges of $\mathcal{T}^{m}$, and no pair of opposite edges in a subtetrahedron of $\mathbf{T}_{p}$ are both in $\mathcal{T}^{m}$. This guarantees that no extra split point is needed for subtetrahedra of $\mathbf{T}_{p}$ in order to satisfy that each face of a subtetrahedron does not have 2 split points, since only edges of $\mathcal{T}^{m}$ may have split points. Therefore, it suffices to apply $\mathrm{SUB}_{2}$ or $\mathrm{SUB}_{4}^{2}$ to subtetrahedra of $\mathbf{T}_{p}$ in step (4) of QLRS.

Now we prove that step (4) of QLRS produces a conforming mesh. It is obvious that any two tetrahedra cannot intersect in their interior. Therefore it suffices to prove that the resulting mesh is conforming on any face. Suppose $F$ is an interior face in a conforming mesh $\mathcal{T}^{m}, m \geq 0$. If $F$ is a regular face, it will have the configuration of Figure 1a, 1c, or $F$ itself at the end of step (4) of QLRS depending on the number of split points on the face, independent of the use of $\mathrm{SUB}_{2}, \mathrm{SUB}_{4}^{1}$, $\mathrm{SUB}_{4}^{2}$, or $\mathrm{SUB}_{8}$ on the tetrahedra sharing $F$. If $F$ is an irregular face, by step (4) 
of QLRS, either $F$ does not change (in this case, the conformity is obvious) or the parents of the tetrahedra sharing $F$ are first subdivided by $\mathrm{SUB}_{8}$ or $\mathrm{SUB}_{4}^{2}$ without producing nonconformity on $F^{\prime}$ 's direct parent $F^{\prime}$, i.e., $F^{\prime}$ is subdivided into the configuration of Figure 1a. Further possible subdivisions on the subfaces of $F^{\prime}$ can be treated just like the case for a regular face, since the subfaces of $F^{\prime}$ are regular. Thus $\mathcal{T}^{m+1}$ is conforming.

Since we mainly use $\mathrm{SUB}_{8}$ in QLRS, Theorem 6 below gives a property similar to that in Theorem 2. A numerical bound on the shape of subtetrahedra produced by QLRS in terms of the tetrahedron shape measure $\eta$ is given in Theorem 7, which needs Lemma 3.

Theorem 6. For any initial mesh $\mathcal{T}$, there are only a finite number, which depends on the number of tetrahedra in $\mathcal{T}$, of classes of similar tetrahedra in all refined tetrahedra generated by QLRS.

Proof. Note that in QLRS, if a tetrahedron is subdivided to $n \geq 1$ levels, $\mathrm{SUB}_{8}$ is applied in the first $n-1$ levels of refinement, and each subtetrahedron is set to have the same subdivision pattern as its parent. At the $n$th level of refinement, $\mathrm{SUB}_{8}, \mathrm{SUB}_{2}, \mathrm{SUB}_{4}^{1}$, or $\mathrm{SUB}_{4}^{2}$ is used. The theorem is established by Theorem 2, since each class of similar tetrahedra in the first $n-1$ levels of refinement produces at most four new classes of similar tetrahedra at the last step of refinement.

Lemma 3. Let $\mathbf{T}_{k}^{1}$ be any subtetrahedron generated by $S U B_{2}, S U B_{4}^{1}$, or $S U B_{4}^{2}$ applied to $\mathbf{T}$. Then

$$
\eta\left(\mathbf{T}_{k}^{1}\right) \geq 2 \sqrt[3]{4} / 11 \eta(\mathbf{T}) .
$$

Proof. Let $\mathbf{t}_{i j}$ denote the midpoint of $\mathbf{t}_{i} \mathbf{t}_{j}$ and $l_{i j}=\left|\mathbf{t}_{i} \mathbf{t}_{j}\right|$. For a triangle $\mathbf{t}_{A} \mathbf{t}_{B} \mathbf{t}_{C}$,

$$
\left|\mathbf{t}_{C} \mathbf{t}_{A B}\right|^{2}=\left(2 l_{A C}^{2}+2 l_{B C}^{2}-l_{A B}^{2}\right) / 4,
$$

and for a tetrahedron with vertices $\mathbf{t}_{0}, \mathbf{t}_{1}, \mathbf{t}_{2}, \mathbf{t}_{3}$,

$$
\begin{aligned}
\left|\mathbf{t}_{03} \mathbf{t}_{12}\right|^{2} & =\left(\mathbf{t}_{0}-\mathbf{t}_{1}+\mathbf{t}_{3}-\mathbf{t}_{2}\right)^{T}\left(\mathbf{t}_{0}-\mathbf{t}_{1}+\mathbf{t}_{3}-\mathbf{t}_{2}\right) / 4 \\
& =\left(l_{01}^{2}+l_{23}^{2}+l_{02}^{2}+l_{13}^{2}-l_{03}^{2}-l_{12}^{2}\right) / 4 .
\end{aligned}
$$

Case 1: $\mathbf{T}_{k}^{1}$ is a subtetrahedron generated by $\mathrm{SUB}_{2}$ (see Figure 4a). By simple algebraic manipulation using (10), (2), and the fact that the volume of $\mathbf{T}_{k}^{1}$ is half the volume of $\mathbf{T}$, it follows that

$$
\frac{\eta\left(\mathbf{T}_{1}^{1}\left(\mathbf{t}_{0}, \mathbf{t}_{1}, \mathbf{t}_{12}, \mathbf{t}_{3}\right)\right)}{\eta(\mathbf{T})}=2 \sqrt[3]{2} \times \frac{\sum_{0 \leq i<j \leq 3} l_{i j}^{2}}{6 l_{01}^{2}+6 l_{13}^{2}+4 l_{03}^{2}+2 l_{23}^{2}+2 l_{02}^{2}-l_{12}^{2}} \geq \sqrt[3]{2} / 3
$$

Similarly, we obtain

$$
\eta\left(\mathbf{T}_{2}^{1}\left(\mathbf{t}_{0}, \mathbf{t}_{2}, \mathbf{t}_{12}, \mathbf{t}_{3}\right)\right) \geq \sqrt[3]{2} / 3 \eta(\mathbf{T}) .
$$

Case 2: $\mathbf{T}_{k}^{1}$ is a subtetrahedron generated by $\mathrm{SUB}_{4}^{1}$ (see Figure $4 \mathrm{~b}$ ). Using (10), (11), and (2), we have

$$
\frac{\eta\left(\mathbf{T}_{1}^{1}\left(\mathbf{t}_{0}, \mathbf{t}_{1}, \mathbf{t}_{12}, \mathbf{t}_{03}\right)\right)}{\eta(\mathbf{T})}=\sqrt[3]{4} \times \frac{\sum_{0 \leq i<j \leq 3} l_{i j}^{2}}{9 l_{01}^{2}+3 l_{13}^{2}+3 l_{02}^{2}+l_{23}^{2}-l_{03}^{2}-l_{12}^{2}} .
$$

By the triangle inequality,

$$
l_{01}^{2} \leq 2\left(l_{03}^{2}+l_{13}^{2}\right) \leq 2\left(l_{03}^{2}+2 l_{12}^{2}+2 l_{23}^{2}\right)
$$




$$
l_{01}^{2} \leq l_{02}^{2}+l_{03}^{2}+l_{12}^{2}+l_{13}^{2} .
$$

Note that $9 l_{01}^{2}=l_{01}^{2}+5 l_{01}^{2} / 2+11 l_{01}^{2} / 2$; if the first two items on the right side of this equality are replaced by (15) and (16), respectively, it follows that the denominator of the right side of (14) is $\leq 11 l_{01}^{2} / 2+11 l_{13}^{2} / 2+11 l_{02}^{2} / 2+11 l_{12}^{2} / 2+5 l_{23}^{2}+7 l_{03}^{2} / 2$. Hence

$$
\frac{\eta\left(\mathbf{T}_{1}^{1}\left(\mathbf{t}_{0}, \mathbf{t}_{1}, \mathbf{t}_{12}, \mathbf{t}_{03}\right)\right)}{\eta(\mathbf{T})} \geq \frac{2 \sqrt[3]{4}}{11} .
$$

Similarly, for the other three subtetrahedra, we obtain the same bound as in (17).

Case 3: $\mathbf{T}_{k}^{1}$ is a subtetrahedron generated by $\mathrm{SUB}_{4}^{2}$ (see Figure 4c). Using similar techniques as above, we obtain

$$
\frac{\eta\left(\mathbf{T}_{1}^{1}\left(\mathbf{t}_{0}, \mathbf{t}_{01}, \mathbf{t}_{02}, \mathbf{t}_{3}\right)\right)}{\eta(\mathbf{T})}=\sqrt[3]{4} \times \frac{\sum_{0 \leq i<j \leq 3} l_{i j}^{2}}{8 l_{03}^{2}+2 l_{13}^{2}+2 l_{23}^{2}+l_{12}^{2}} \geq \frac{\sqrt[3]{4}}{5},
$$

since $3 l_{03}^{2} \leq 3 l_{01}^{2}+3 l_{02}^{2}+3 l_{13}^{2}+3 l_{23}^{2}$. For the other two subtetrahedra in the corners, we obtain the same bound as in (18). For the subtetrahedron in the middle, we have

$$
\frac{\eta\left(\mathbf{T}_{4}^{1}\left(\mathbf{t}_{01}, \mathbf{t}_{12}, \mathbf{t}_{02}, \mathbf{t}_{3}\right)\right)}{\eta(\mathbf{T})}=\sqrt[3]{4} \times \frac{\sum_{0 \leq i<j \leq 3} l_{i j}^{2}}{4 l_{03}^{2}+4 l_{13}^{2}+4 l_{23}^{2}} \geq \frac{\sqrt[3]{4}}{4} .
$$

Combining (12), (13), (17), (18), and (19) yields (9).

Theorem 7. If $\mathbf{T}_{i}^{n}$ is a refined tetrahedron of any tetrahedron $\mathbf{T}$ in $\mathcal{T}$, produced by $Q L R S$, then

$$
\eta\left(\mathbf{T}_{i}^{n}\right) \geq c \eta(\mathbf{T})
$$

where $c=\sqrt[3]{4} / 11 \approx 0.1443$.

Proof. If $\mathbf{T}_{i}^{n}$ is of type $\mathrm{S}_{8}$, then (20) follows from (3). Suppose $\mathbf{T}_{i}^{n}$ is of type $\mathrm{S}_{2}$, $\mathrm{S}_{4}^{1}$, or $\mathrm{S}_{4}^{2}$, and $\mathbf{T}_{j}^{n-1}$ is the direct parent of $\mathbf{T}_{i}^{n}, n \geq 1$. According to QLRS, $\mathbf{T}_{j}^{n-1}$ is produced by repeatedly applying $\mathrm{SUB}_{8}$ to $\mathbf{T}$. By Theorem 3,

$$
\eta\left(\mathbf{T}_{j}^{n-1}\right) \geq 0.5 \eta(\mathbf{T}) \text {. }
$$

By Lemma 3,

$$
\eta\left(\mathbf{T}_{i}^{n}\right) \geq 2 \sqrt[3]{4} / 11 \eta\left(\mathbf{T}_{j}^{n-1}\right)
$$

Combining (21) and (22) yields (20).

For any tetrahedron $\mathbf{T}$ in $\mathcal{T}^{m}, m \geq 0$, we recursively define the subdivision level of $\mathbf{T}$, denoted by $\ell(\mathbf{T})$, as follows. If $\mathbf{T}$ is a tetrahedron of the initial mesh $\mathcal{T}$, $\ell(\mathbf{T})=0$; otherwise, $\ell(\mathbf{T})=\ell\left(\mathbf{T}_{p}\right)+3, \ell\left(\mathbf{T}_{p}\right)+2$, or $\ell\left(\mathbf{T}_{p}\right)+1$ if $\mathbf{T}$ is a subtetrahedron produced by applying $\mathrm{SUB}_{8}, \mathrm{SUB}_{4}^{1}$ or $\mathrm{SUB}_{4}^{2}$, or $\mathrm{SUB}_{2}$ to $\mathbf{T}_{p}$, respectively.

Theorem 8. For any interior face shared by two adjacent tetrahedra $\mathbf{T}_{L}$ and $\mathbf{T}_{R}$ in $\mathcal{T}^{m}, m \geq 0$, generated by $Q L R S,\left|\ell\left(\mathbf{T}_{L}\right)-\ell\left(\mathbf{T}_{R}\right)\right| \leq 2$, and the upper bound is tight.

Proof. Let $F$ be an interior face shared by two adjacent tetrahedra $\mathbf{T}_{L}$ and $\mathbf{T}_{R}$ in $\mathcal{T}^{m}, m \geq 0$. We first prove by contradiction that if $\mathbf{T}_{L}$ and $\mathbf{T}_{R}$ are tetrahedra of type $\mathrm{S}_{8}, \ell\left(\mathbf{T}_{L}\right)=\ell\left(\mathbf{T}_{R}\right)$. Let $\ell\left(\mathbf{T}_{L}\right)=3 p, \ell\left(\mathbf{T}_{R}\right)=3 q<3 p$, and $0<r \leq q$. Note that when $\mathrm{SUB}_{8}$ is applied to a tetrahedron, each face of the tetrahedron is subdivided. Therefore, for any $r$, the ancestor $\hat{\mathbf{T}}_{L}$ of $\mathbf{T}_{L}$ at level $3 p-3 r$ must have 
a common face with the ancestor $\hat{\mathbf{T}}_{R}$ of $\mathbf{T}_{R}$ at level $3 q-3 r$. Let $r=q$. Then $\hat{\mathbf{T}}_{R}$ is a tetrahedron in the initial mesh, but $\hat{\mathbf{T}}_{L}$ is a tetrahedron at level $3(p-q)$. These two tetrahedra cannot share a common face, a contradiction.

Suppose one of the two tetrahedra, $\mathbf{T}_{L}$ say, is not a tetrahedron of type $\mathrm{S}_{8}$. If $\mathbf{T}_{R}$ is a tetrahedron of type $\mathrm{S}_{8}$, then $F$ must be like face $\mathbf{t}_{0} \mathbf{t}_{1} \mathbf{t}_{3}\left(\right.$ or $\left.\mathbf{t}_{0} \mathbf{t}_{2} \mathbf{t}_{3}\right)$ in Figure $4 \mathrm{a}$, or a subface of $\mathbf{t}_{0} \mathbf{t}_{1} \mathbf{t}_{2}$ in Figure $4 \mathrm{c}$. In the former case, $\ell\left(\mathbf{T}_{R}\right)=\ell\left(\mathbf{T}_{L}\right)-1$. In the latter case, $\ell\left(\mathbf{T}_{R}\right)=\ell\left(\mathbf{T}_{L}\right)+1$.

Now suppose $\mathbf{T}_{R}$ is also not a tetrahedron of type $\mathrm{S}_{8}$. First, if $F$ is an irregular face, $\ell\left(\mathbf{T}_{L}\right)=\ell\left(\hat{\mathbf{T}}_{L}\right)+1$ or $\ell\left(\hat{\mathbf{T}}_{L}\right)+2$, and $\ell\left(\mathbf{T}_{R}\right)=\ell\left(\hat{\mathbf{T}}_{R}\right)+1$ or $\ell\left(\hat{\mathbf{T}}_{R}\right)+2$, where $\hat{\mathbf{T}}_{L}$ and $\hat{\mathbf{T}}_{R}$ are $\mathbf{T}_{L}$ and $\mathbf{T}_{R}$ 's parents, respectively. So, $\left|\ell\left(\mathbf{T}_{L}\right)-\ell\left(\mathbf{T}_{R}\right)\right| \leq 1$, since $\ell\left(\hat{\mathbf{T}}_{R}\right)=\ell\left(\hat{\mathbf{T}}_{L}\right)$ by the discussion in the first paragraph. Next, if $F$ is a regular face, the types of $\mathbf{T}_{L}$ and $\mathbf{T}_{R}$ can be one of the following pairs $\left(\mathrm{S}_{2}, \mathrm{~S}_{2}\right),\left(\mathrm{S}_{4}^{2}, \mathrm{~S}_{4}^{2}\right)$, and $\left(\mathrm{S}_{2}, \mathrm{~S}_{4}^{2}\right)$. For the first two pairs, $\ell\left(\mathbf{T}_{L}\right)=\ell\left(\mathbf{T}_{R}\right)$. For the final pair, suppose $\mathbf{T}_{L}$ is a tetrahedron of type $\mathrm{S}_{2}$, and $\mathbf{T}_{R}$ is a tetrahedron of type $\mathrm{S}_{4}^{2}$. Then the only possible situation is that the parent $\hat{\mathbf{T}}_{R}$ of $\mathbf{T}_{R}$ has a common face with the direct grandparent $\hat{\mathbf{T}}_{L}$ of $\mathbf{T}_{L}$. Since $\ell\left(\mathbf{T}_{R}\right)=\ell\left(\hat{\mathbf{T}}_{R}\right)+2, \ell\left(\mathbf{T}_{L}\right)=\ell\left(\hat{\mathbf{T}}_{L}\right)+3+1$, and $\ell\left(\hat{\mathbf{T}}_{R}\right)=\ell\left(\hat{\mathbf{T}}_{L}\right)$, it follows that $\ell\left(\mathbf{T}_{L}\right)=\ell\left(\mathbf{T}_{R}\right)+2$.

Note that if the maximum subdivision level of a tetrahedron in $\mathcal{T}^{k}$ is $\leq 3 k$, then the maximum subdivision level of a tetrahedron in $\mathcal{T}^{k+1}$ is $\leq 3(k+1)$, since each subtetrahedron of a tetrahedron of type $\mathrm{S}_{8}$ in $\mathcal{T}^{k}$ has subdivision level at most $3 k+3$ in $\mathcal{T}^{k+1}$, and each subtetrahedron of the parent of a tetrahedron of type $\mathrm{S}_{2}, \mathrm{~S}_{4}^{1}$, or $\mathrm{S}_{4}^{2}$ in $\mathcal{T}^{k}$ has subdivision level at most $3(k-1)+3+2$ in $\mathcal{T}^{k+1}$. So, by induction on $k$, the maximum subdivision level of a tetrahedron in any $\mathcal{T}^{m}$ is not greater than $3 m, m \geq 0$.

\section{ExPerimental RESUlts}

In QLRS, the main operation is to determine the number of split points for each tetrahedron subject to the condition that each regular face has 0,1 , or 3 split points and each irregular face has 0,1 , or 2 split points. When a split point is added to an edge $e$, each regular face incident on $e$ is checked to decide whether an extra split point is needed for this face. Therefore, the data structures for QLRS should support a fast operation that reports all faces incident on an edge, which can be done by recording the adjacent tetrahedron for each interior face of a tetrahedron. In addition, determining whether an edge has a split point should also be implemented efficiently. To this end, we have designed a set of data structures similar to those in [12], in which a hash table and a stack are used to store all edges with a split point and all tetrahedra needing subdivision at each step of refinement, respectively, and have implemented QLRS in an expected time complexity that is linearly related to the number of refined tetrahedra in a refined mesh (see details in $[9])$.

We report our experimental results for four single tetrahedra (Tables I-IV in [20]) and two tetrahedral meshes of polyhedral regions (one is a convex polyhedron, Figure 1a in [5]; the other is a U-shaped region, Figure 10 in [6]). For a single tetrahedron, we refine all tetrahedra in the mesh at each step of refinement as in $[20,12]$. For the two tetrahedral meshes, a fixed point on the object is chosen as the center of a sphere; at each step of refinement, we refine any tetrahedron with at least one of its vertices in the sphere, and reduce the radius of the sphere by a 
TABLe 1. Problems 1 to 4

\begin{tabular}{|c|c|c|c|c|c|c|c|c|c|c|c|}
\hline \multicolumn{3}{|c|}{$\begin{array}{c}\mathrm{P} 1 \\
\eta=0.8846\end{array}$} & \multicolumn{3}{|c|}{$\begin{array}{c}\mathrm{P} 2 \\
\eta=0.8399\end{array}$} & \multicolumn{3}{|c|}{$\begin{array}{c}\mathrm{P} 3 \\
\eta=0.2835\end{array}$} & \multicolumn{3}{|c|}{$\begin{array}{c}\mathrm{P} 4 \\
\eta=1.0000\end{array}$} \\
\hline 0.0 & 0.0 & 0.0 & 0.0 & 0.0 & 0.0 & 0.0 & 0.0 & 0.0 & 0.0 & 0.0 & 0.0 \\
\hline 4.0 & 2.0 & 2.0 & 4.0 & 0.0 & 0.0 & 0.5 & 0.0 & 0.0 & $2 \sqrt{3}$ & 0.0 & 0.0 \\
\hline 1.0 & 5.0 & 0.0 & 0.0 & 4.0 & 0.0 & 1.5 & 5.0 & 2.0 & $\sqrt{3}$ & 3.0 & 0.0 \\
\hline 0.5 & 0.5 & 5.0 & 0.0 & 0.0 & 4.0 & 0.5 & 0.5 & 5.0 & $\sqrt{3}$ & 1.0 & $2 \sqrt{2}$ \\
\hline
\end{tabular}

TABLE 2. Experimental results for Problems 1, 2

\begin{tabular}{|c|cccrc|ccccc||}
\hline & \multicolumn{3}{|c}{ Problem 1} & \multicolumn{4}{c|}{ Problem 2} \\
level & NTET & $\eta_{\text {min }}$ & $\eta_{\text {ave }}$ & $\eta<0.5$ & $\eta \geq 0.7$ & NTET & $\eta_{\text {min }}$ & $\eta_{\text {ave }}$ & $\eta<0.5$ & $\eta \geq 0.7$ \\
\hline 0 & 1 & 0.8846 & 0.8846 & 0.00 & 100.00 & 1 & 0.8399 & 0.8399 & 0.00 & 100.00 \\
1 & 8 & 0.8664 & 0.9069 & 0.00 & 100.00 & 8 & 0.6872 & 0.7808 & 0.00 & 75.00 \\
2 & 64 & 0.8664 & 0.9124 & 0.00 & 100.00 & 64 & 0.6872 & 0.7660 & 0.00 & 68.75 \\
3 & 512 & 0.8664 & 0.9138 & 0.00 & 100.00 & 512 & 0.6872 & 0.7623 & 0.00 & 67.19 \\
\hline
\end{tabular}

TABLE 3. Experimental results for Problems 3, 4

\begin{tabular}{|c|ccccc|ccccc||}
\hline & \multicolumn{3}{|c}{ Problem 3 } & \multicolumn{4}{c|}{ Problem 4} \\
level & NTET & $\eta_{\text {min }}$ & $\eta_{\text {ave }}$ & $\eta<0.2$ & $\eta \geq 0.4$ & NTET & $\eta_{\min }$ & $\eta_{\text {ave }}$ & $\eta<0.5$ & $\eta \geq 0.7$ \\
\hline 0 & 1 & 0.2835 & 0.2835 & 0.00 & 0.00 & 1 & 1.0000 & 1.0000 & 0.00 & 100.00 \\
1 & 8 & 0.2756 & 0.2819 & 0.00 & 0.00 & 8 & 0.8571 & 0.9286 & 0.00 & 100.00 \\
2 & 64 & 0.2756 & 0.2815 & 0.00 & 0.00 & 64 & 0.8571 & 0.9107 & 0.00 & 100.00 \\
3 & 512 & 0.2756 & 0.2814 & 0.00 & 0.00 & 512 & 0.8571 & 0.9062 & 0.00 & 100.00 \\
\hline
\end{tabular}

factor. The quantities used to measure the quality at each level of refinement are the number of tetrahedra NTET, the minimum mean ratio $\eta_{\min }$ (over all tetrahedra), the average mean ratio $\eta_{\text {ave }}$, and the percentage of tetrahedra whose $\eta$ value is less/greater than a certain number. The mean ratio $\eta(0<\eta \leq 1)$ approaches zero or the maximum value for a poorly-shaped or well-shaped tetrahedron, respectively. In our experiments, a tetrahedron with $\eta<0.3$ (or $\eta \geq 0.7$ ) is considered poorly shaped (or well shaped). The reason for using $\eta$ to report our experimental results is because our theoretical results are based on it. By the "equivalence" of tetrahedron shape measures [11], i.e., $c_{0} \mu^{e_{0}} \leq \nu \leq c_{1} \mu^{e_{1}}$, where $\mu, \nu$ are shape measures and $c_{0}, c_{1}, e_{0}, e_{1}$ are positive constants, we can expect (and have observed) similar statistical results based on other tetrahedron shape measures, since a larger or smaller measure value for a tetrahedron indicates that the tetrahedron is well shaped or poorly shaped, respectively.

The four single tetrahedra are listed in Table 1 in terms of the coordinates of the four vertices. P1 and P2 are well-shaped tetrahedra; P3 is a poorly-shaped tetrahedron; $\mathrm{P} 4$ is the regular tetrahedron, where $\sqrt{3}$ and $\sqrt{2}$ are rounded to 16 decimal places. For the two polyhedral regions, by using the methods described in $[5,6]$, the convex polyhedron is subdivided into 273 tetrahedra, and has minimum mean ratio 0.6230 after local transformations are used to obtain an improved-quality mesh with respect to radius ratio $\rho$; the U-shaped object is subdivided into 466 tetrahedra with minimum mean ratio 0.5580 after local transformations are performed.

Tables 2 to 4 show experimental results based on QLRS. The results seem to confirm that $\eta_{\min }$ and $\eta_{\text {ave }}$ converge asymptotically to a fixed value for QLRS, and the experimental results are much better than the theoretical estimate of the constant $c$ in Theorem 7 (since we do not have a tight bound). 
TABLE 4. Experimental results for two tetrahedral meshes

\begin{tabular}{|c|rcrrr|rrrrr|}
\hline & \multicolumn{9}{|c|}{ Convex Polyhedron } & \multicolumn{4}{c|}{ U-shaped Object } \\
level & NTET & $\eta_{\text {min }}$ & $\eta_{\text {ave }}$ & $\eta<0.5$ & $\eta \geq 0.7$ & NTET & $\eta_{\min }$ & $\eta_{\text {ave }}$ & $\eta<0.5$ & $\eta \geq 0.7$ \\
\hline 1 & 273 & 0.6230 & 0.8185 & 0.00 & 92.67 & 466 & 0.5580 & 0.7651 & 0.00 & 80.47 \\
2 & 419 & 0.4265 & 0.7790 & 2.63 & 80.91 & 588 & 0.4373 & 0.7628 & 1.19 & 79.25 \\
3 & 1343 & 0.3729 & 0.7611 & 5.10 & 79.12 & 931 & 0.3957 & 0.7514 & 3.33 & 74.33 \\
4 & 1830 & 0.3729 & 0.7616 & 4.24 & 78.38 & 1494 & 0.3877 & 0.7430 & 4.95 & 73.09 \\
\hline
\end{tabular}

TABle 5. Performance on the expansion of refinement

\begin{tabular}{||r|rrrrr|rrrrr||}
\hline & \multicolumn{9}{|c|}{ Convex Polyhedron } & \multicolumn{4}{c|}{ U-shaped Object } \\
\hline NTETC & 15 & 38 & 62 & 47 & 24 & 14 & 39 & 64 & 57 & 41 \\
NTETR & 38 & 116 & 121 & 100 & 54 & 26 & 76 & 123 & 116 & 87 \\
NTETR/NTETC & 2.53 & 3.05 & 1.95 & 2.13 & 2.25 & 1.86 & 1.95 & 1.92 & 2.04 & 2.12 \\
NTET & 273 & 419 & 843 & 1392 & 1830 & 466 & 588 & 931 & 1494 & 2020 \\
\hline
\end{tabular}

TABLE 6. Improved-quality meshes for two polyhedral regions

\begin{tabular}{|c|rrrrr|rrrrr||}
\hline & \multicolumn{9}{|c|}{ Convex Polyhedron } & \multicolumn{4}{c|}{ U-shaped Object } \\
level & NTET & $\eta_{\text {min }}$ & $\eta_{\text {ave }}$ & $\eta<0.5$ & $\eta \geq 0.7$ & NTET & $\eta_{\min }$ & $\eta_{\text {ave }}$ & $\eta<0.5$ & $\eta \geq 0.7$ \\
\hline 0 & 271 & 0.6230 & 0.8203 & 0.00 & 92.62 & 464 & 0.5825 & 0.7664 & 0.00 & 80.17 \\
1 & 415 & 0.4297 & 0.7804 & 2.41 & 82.41 & 586 & 0.5554 & 0.7688 & 0.00 & 79.86 \\
2 & 838 & 0.4297 & 0.7735 & 2.15 & 81.62 & 929 & 0.4458 & 0.7611 & 0.75 & 76.21 \\
3 & 1388 & 0.4297 & 0.7710 & 1.51 & 80.04 & 1491 & 0.4348 & 0.7536 & 1.27 & 73.91 \\
4 & 1825 & 0.4297 & 0.7700 & 1.15 & 79.01 & 2014 & 0.4159 & 0.7478 & 1.84 & 72.44 \\
\hline
\end{tabular}

Let NTETC and NTETR denote the number of tetrahedra chosen for refinement and the number of tetrahedra actually refined, respectively, at each step of refinement. The ratio NTETR/NTETC reflects the expansion of refinement at a local region. Table 5 lists NTETC, NTETR, NTETR/NTETC, and NTET for the two polyhedral regions. At each step of refinement, we choose a small number of tetrahedra around a fixed point for refinement. It seems that the ratio NTETR/NTETC is bounded above by a small constant (i.e., NTETR does not expand rapidly against NTETC), which is what we expect for "local" refinement.

Similar to [12], local transformations [4], which are based on two different triangulations of certain configurations of five distinct noncoplanar 3-D points (see Figure 6), can be applied with respect to some criteria, such as locally improving the minimum tetrahedron shape measure value. The algorithms in $[4,7]$ can be used to improve the quality of tetrahedra towards an optimal mesh with respect to $\eta$. To keep the properties in Theorems 6 and 7, we only apply local transformations to the final refined mesh, i.e., no refinement is applied to meshes improved by local transformations. The statistical results for the improved-quality meshes of the two polyhedral regions are provided in Table 6 . The improvements are not very significant, compared with the improved results in [12]. This may imply that QLRS produces tetrahedra of relatively good shape, compared with local refinement based on bisection. 


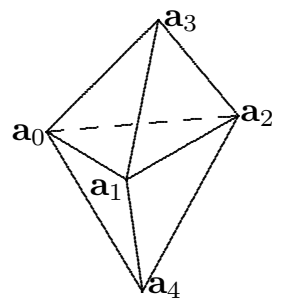

(a)

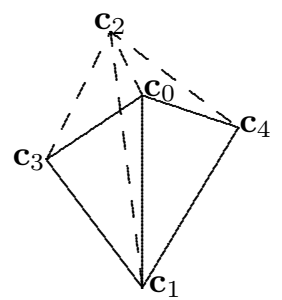

(c)

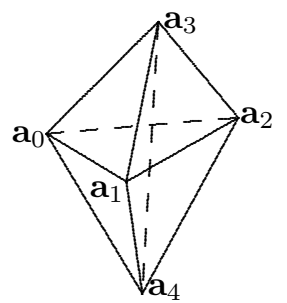

(b)

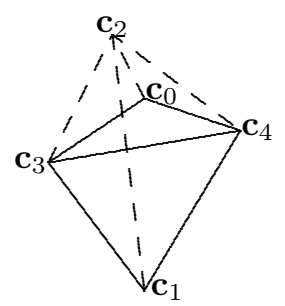

(d)

FIGURE 6. The first type of local transformation replaces the 2 tetrahedra $\mathbf{A}_{1}\left(\mathbf{a}_{0}, \mathbf{a}_{1}, \mathbf{a}_{2}, \mathbf{a}_{3}\right), \mathbf{A}_{2}\left(\mathbf{a}_{0}, \mathbf{a}_{1}, \mathbf{a}_{2}, \mathbf{a}_{4}\right)$ in (a) by the 3 tetrahedra $\mathbf{A}_{3}\left(\mathbf{a}_{0}, \mathbf{a}_{1}, \mathbf{a}_{3}, \mathbf{a}_{4}\right), \mathbf{A}_{4}\left(\mathbf{a}_{0}, \mathbf{a}_{2}, \mathbf{a}_{3}, \mathbf{a}_{4}\right), \mathbf{A}_{5}\left(\mathbf{a}_{1}, \mathbf{a}_{2}, \mathbf{a}_{3}, \mathbf{a}_{4}\right)$ in (b), or vice versa, where no 4 of the 5 vertices $\mathbf{a}_{i}$ are coplanar and line segment $\mathbf{a}_{3} \mathbf{a}_{4}$ intersects the interior of triangle $\mathbf{a}_{0} \mathbf{a}_{1} \mathbf{a}_{2}$. The second type of local transformation replaces the 2 tetrahedra $\mathbf{C}_{1}\left(\mathbf{c}_{0}, \mathbf{c}_{1}, \mathbf{c}_{2}, \mathbf{c}_{3}\right), \mathbf{C}_{2}\left(\mathbf{c}_{0}, \mathbf{c}_{1}, \mathbf{c}_{2}, \mathbf{c}_{4}\right)$ in (c) by the 2 tetrahedra $\mathbf{C}_{3}\left(\mathbf{c}_{0}, \mathbf{c}_{2}, \mathbf{c}_{3}, \mathbf{c}_{4}\right), \mathbf{C}_{4}\left(\mathbf{c}_{1}, \mathbf{c}_{2}, \mathbf{c}_{3}, \mathbf{c}_{4}\right)$ in (d), or vice versa, where $\mathbf{c}_{0} \mathbf{c}_{3} \mathbf{c}_{1} \mathbf{c}_{4}$ is a planar convex quadrilateral and vertex $\mathbf{c}_{2}$ is not coplanar with the other 4 vertices

\section{Concluding Remarks}

We have presented a local refinement algorithm based mainly on an 8-subtetrahedron subdivision, and have shown that the algorithm produces guaranteed-quality meshes. Experimental results show that the number of tetrahedra actually refined in order to keep a conforming mesh is limited by a small number times the number of tetrahedra chosen for refinement, which implies that the algorithm is truly local. The quality of refined meshes for various examples show a very consistent and satisfactory performance.

The algorithm presented here is preferable if the mesh in a refined region is relatively coarse; otherwise, the one described in [12] based on a bisection procedure may be better. It is worth considering the situation that QLRS is applied to some parts of a refined region while the algorithm in [12] is used in other parts. A critical problem is to ensure a conforming mesh with guaranteed-quality when the expansion of refinement from different parts meet each another.

After submitting this paper, we discovered that Zhang's thesis [21] contains some results similar to those in $\S 2$. In particular, [21] contains a longer proof of Theorem 2 and a version of Theorem 3 with a different shape measure and a nontight lower bound. 


\section{REFERENCES}

1. R. E. Bank (1990), PLTMG: A Software Package for Solving Elliptic Partial Differential Equations: Users' Guide 6.0, SIAM, Philadelphia. CMP 90:12

2. R. E. Bank and A. H. Sherman (1981), An adaptive multilevel method for elliptic boundary value problems, Computing, 29, pp. 91-105. MR 83c:65229

3. G. H. Golub and C. F. Van Loan (1989), Matrix Computations, 2nd edition, Johns Hopkins University Press, Baltimore. MR 90d:65055

4. B. Joe (1989), Three-dimensional triangulations from local transformations, SIAM J. Sci. Stat. Comput., 10, pp. 718-741. MR 91c:65075

5. B. Joe (1991), Delaunay versus max-min solid angle triangulations for three-dimensional mesh generation, Intern. J. Num. Meth. Eng., 31, pp. 987-997.

6. B. Joe (1994), Tetrahedral mesh generation in polyhedral regions based on convex polyhedron decompositions, Intern. J. Num. Meth. Eng., 37, pp. 693-713. CMP 94:07

7. B. Joe (1995), Construction of three-dimensional improved-quality triangulations using local transformations, SIAM J. Sci. Comput., 16, 1292-1307. CMP 96:02

8. B. Kearfott (1978), A proof of convergence and an error bound for the method of bisection in $\mathbf{R}^{n}$, Math. Comp., 32, pp. 1147-1153. MR 58:13677

9. A. Liu (1994), Quality local refinement of tetrahedral meshes, Ph.D. Thesis, Department of Computing Science, University of Alberta.

10. A. Liu and B. Joe (1994), On the shape of tetrahedra from bisection, Math. Comp., 63, pp. 141-154. MR 94j:65113

11. A. Liu and B. Joe (1994), Relationship between tetrahedron shape measures, BIT, 34, pp. 268-287.

12. A. Liu and B. Joe (1995), Quality local refinement of tetrahedral meshes based on bisection, SIAM J. Sci. Comput., 16, 1269-1291. CMP 96:02

13. R. Löhner, K. Morgan, and O. C. Zienkiewicz (1986), Adaptive grid refinement for the compressible Euler equations, in I. Babuška et al., Accuracy Estimates and Adaptive Refinements in Finite Element Computations, Wiley, New York. CMP 19:09

14. W. F. Mitchell (1989), A comparison of adaptive refinement techniques for elliptic problems, ACM Trans. on Mathematical Software, 15, pp. 326-347. CMP 90:15

15. D. Moore and J. Warren (1990), Adaptive mesh generation I: packing space, Technical Report, Department of Computer Science, Rice University.

16. T. W. Nehl and D. A. Field (1991), Adaptive refinement of first order tetrahedral meshes for magnetostatics using local Delaunay subdivisions, IEEE Trans. on Magnetics, 27, pp. 4193-4196.

17. M.-C. Rivara (1984), Algorithms for refining triangular grids suitable for adaptive and multigrid techniques, Intern. J. Num. Meth. Eng., 20, pp. 745-756. MR 85h:65258

18. M.-C. Rivara (1987), A grid generator based on 4-triangles conforming mesh-refinement algorithms, Intern. J. Num. Meth. Eng., 24, pp. 1343-1354.

19. M.-C. Rivara (1989), Selective refinement/derefinement algorithms for sequences of nested triangulations, Intern. J. Num. Meth. Eng., 28, pp. 2889-2906. MR 90j:57018

20. M.-C. Rivara and C. Levin (1992), A 3-D refinement algorithm suitable for adaptive and multi-grid techniques, Comm. Appl. Num. Meth., 8, pp. 281-290.

21. S. Zhang (1988), Multi-level iterative techniques, Ph.D. Thesis, Department of Mathematics, Pennsylvania State University.

Department of Computing Science, University of Alberta, Edmonton, Alberta, Canada T6G 2H1

E-mail address: anwei@cs.ualberta.ca

E-mail address: barry@cs.ualberta.ca 